\title{
Trastorno por Déficit de Atención e Hiperactividad (TDAH): Prevalencia y Características Sociodemográficas en Población Reclusa
}

\author{
Attention Deficit Hyperactivity Disorder (ADHD): Prevalence \\ and Sociodemographic Features in Imprisoned Population
}

\author{
Celestino Rodríguez Pérez ${ }^{a}$, José Carlos Núñez Pérez ${ }^{a}$, Francisco Javier Rodríguez Díaz ${ }^{*}$, \\ Alba Parrales Granda ${ }^{a}$, Carolina Bringas Molleda ${ }^{b} \&$ Trinidad García Fernández ${ }^{a}$ \\ ${ }^{a}$ Universidad de Oviedo, Oviedo, España \& ${ }^{b}$ Universidad de Extremadura, Cáceres, España
}

\begin{abstract}
Resumen
Diversos estudios coinciden en señalar que la prevalencia de enfermedades mentales en internos penitenciarios es significativamente mayor que en la población general. En este contexto, la literatura muestra que existe una importante presencia de síntomas del Trastorno por Déficit de Atención con Hiperactividad (TDAH) en esta población. Sin embargo, la investigación al respecto es escasa. En este estudio se estimó la prevalencia con cribado positivo para TDAH en la población penitenciaria, y se establecieron las características sociodemográficas asociadas al trastorno. Se administraron dos escalas de evaluación del TDAH ampliamente empleadas y validadas en población adulta - ASRS y WURS. Los participantes fueron 143 presos del Establecimiento Penitenciario de Villabona (Asturias, España), con un rango de edad entre 18 y 69 años. Los resultados obtenidos indican una prevalencia aparente del TDAH en torno al $25 \%$ utilizando ambas escalas y con resultados coincidentes entre ellas. Además se encontraron diferencias significativas por sexo, por nivel de escolaridad y situación laboral, con diferentes perfiles según la presencia de los síntomas del TDAH. Estos resultados evidencian la necesidad de estudiar las consecuencias de este trastorno en la población penitenciaria, con el objeto de mejorar el manejo terapéutico en este contexto, atendiendo a sus características. Palabras clave: TDAH, prisión, prevalencia, características sociodemográficas, evaluación de la conducta.
\end{abstract}

\begin{abstract}
There is a good deal of evidence suggesting that the prevalence of mental disorders among imprisoned people is significantly higher that in general population. In this context, literature shows that there is an important presence of Attention Deficit Hyperactivity Disorder (ADHD) within this population. However, research on this topic is lacking. This study estimated the prevalence of ADHD in a penitentiary population by means of positive screening and established the sociodemographic characteristics related to the presence of the disorder. Two different scales of ADHD, widely applied and validated for adult population, were used for this purpose - ASRS and WURS. One-hundred and forty three prisoners between 18 and 69 years old from Villabona's Penitentiary Establishment (Asturias, Spain) took part in this study. Results showed 25\% of apparent prevalence of ADHD, using both scales. Statistically significant differences were found in sex, educational level and employment situation, showing different profiles as a function of the presence of ADHD symptoms. These results highlight the need to study the consequences of this disorder within imprisoned populations in order to improve therapeutic management, paying attention to their characteristics.

Keywords: ADHD, Prison, Prevalence, Sociodemographic characteristics, Behavior assessment.
\end{abstract}

La prevalencia del Trastorno por Déficit de Atención e Hiperactividad -en adelante TDAH- (American Psychiatric Association [APA], 2000) se estima entre el 3 y el 7\%, afectando en mayor medida al género masculino que al

*Dirección postal: Universidad de Oviedo, Departamento de Psicología, Facultad de Psicología, Plaza Feijóo, s/n, Despacho 215, Oviedo, España 33003. E-mail: gallego@ uniovi.es femenino, en una proporción que varía de 2 a 1 hasta 9 a 1 según diferentes investigaciones (Caballo \& Simón, 2001), teniendo que ser considerada estas proporciones de género en los diferentes estudios sobre TDAH en adultos y las conclusiones aportadas (Rösler et al., 2004). La evolución de los síntomas es dependiente de la sintomatología en la infancia, de modo que los síntomas de hiperactividadimpulsividad durante la edad escolar tienden a disminuir en la adolescencia; sin embargo, cuando los síntomas están 
Pérez, C. R., Pérez, J. C. N., Díaz, F. J. R., Granda, A. P., Molleda C. B. \& Fernández, T. G. (2015). Trastorno por Déficit de Atención e Hiperactividad (TDAH): Prevalencia y Características Sociodemográficas en Población Reclusa.

relacionados con la inatención, éstos persisten incluso hasta la edad adulta (Brown, 2003).

Por otro lado, y en concreto en la edad adulta, las enfermedades mentales parecen ser más frecuentes en población penitenciaria que en población general (Fazel \& Seewald, 2012; Martin, Wamboldt, O'Connor, Fortier, \& Simpson, 2013). El informe de prevalencia de trastornos mentales en centros penitenciarios españoles (Vicens-Pons \& Grupo de Prevalencia en Cárceles [Grupo PRECA], 2009) recoge que ocho de cada diez internos ingresados en cárceles han padecido a lo largo de su vida un trastorno mental, con un $40 \%$ de estos internos presentando alguno de ellos en la actualidad -alrededor del $80 \%$ presenta historia de trastorno por uso de sustancias, principalmente alcohol y cocaína. Varios estudios han encontrado una prevalencia de enfermedades mentales cinco veces mayor en internos penitenciarios que en la población general (Fazel \& Seewald, 2012; Martin et al., 2013; Sanz-García, Dueñas, $\&$ Muro, 2010).

No obstante, cabe resaltar la escasa investigación del TDAH en este grupo de interno, al menos en comparación con la investigación dedicada a este trastorno en otro tipo de poblaciones. Esta realidad se constata en el número de trabajos publicados. Así, por ejemplo en Europa, 22.177 artículos fueron publicados sobre el TDAH / TDA en Embase y Medline entre 1974 y 2012; de éstos, solamente 5.591 estudiaban el TDAH en adultos. En total, 237 publicaciones fueron consideradas clínicamente relevantes (Ramos-Quiroga, Montoya, Kutzelnigg, Deberdt, \& Sobanski, 2013), cubriendo gran variedad de temas como el tratamiento del TDAH, su comorbilidad, y estudios de neuro-imagen. Son muy pocos, sin embargo, los trabajos relacionados con la población reclusa (Ginsberg, Hirvikoski, \& Lindefors, 2010). En este sentido, através de la base de datos de PsycInfo la búsqueda bibliográfica realizada con la palabra clave ADHD agrupa 8983 referencias de artículos publicados hasta 2013 inclusive. La primera publicación que aparece es de 1988, dándose un crecimiento sostenido y posteriormente realzado a partir de 2005 -ofrece un porcentaje superior al $65 \%$ de la producción total, es decir, 5990 referencias de artículos. Al añadir a esta búsqueda la palabra clave para la población de nuestro estudio ('prisoners') tan solo se obtienen 21 artículos, publicados desde 2007 a 2012. Estos estudios ofrecen una prevalencia del trastorno en prisión que abarca un rango amplio, de un 10 hasta un $70 \%$ (Ghanizadeh, Mohammadi, Akhondzadeh, \& Sanaei-Zadeh, 2011). Asimismo, se observa que la presencia del TDAH aparece frecuentemente asociado a otros trastornos, actuando como factor de riesgo para la conducta criminal y antisocial, además de predecir el futuro consumo de sustancias (Einat \& Einat, 2008; Ghanizadeh et al., 2011; Rösler et al., 2004).

Dada esta realidad, establecer la prevalencia del TDAH en población penitenciaria cobra especial relevancia en el momento actual (Vegue-González, Álvaro-
Brun, Santiago-Sáez, \& Kanaan-Kanaan, 2011). Además, se puede constatar que, por un lado, es resaltable su comorbilidad con desordenes tales como dificultades de aprendizaje, depresión y ansiedad, trastorno oposicionista desafiante, trastorno de ansiedad y trastornos del humor (Brown et al., 2001; Schatz \& Rostain, 2006; Spencer et al., 2006); y por otro lado, esta realidad se conforma con una mayor presencia de desórdenes mentales en población penitenciaria que en población general (Fazel \& Seewald, 2012; Ghanizadeh, Nouri, \& Nabr, 2012; Martin et al., 2013).

Asimismo, estos resultados confirman una presencia del TDAH en prisión más elevada que en población general (Einat \& Einat, 2008; Ghanizadeh et al., 2011; Rösler et al., 2004), destacando en la población reclusa con TDAH un control de impulsos bajo y un riesgo de reincidencia elevado entre los internos (Eme, 2008), la cual se incrementa hasta cinco veces más si el preso ha cumplido condena en más de una ocasión (Vicens-Pons \& Grupo PRECA, 2009). En esta línea, el estudio de Sanz-García et al. (2010), realizado con internos en tratamiento psiquiátrico en el medio penitenciario, refiere que aquellos con TDAH delinquen a más temprana edad, con una media de comisión del primer delito a los 22 años frente a los 29 años del resto de reclusos con otras patologías psiquiátricas. Estos también cometen mayor número de delitos, con una media de 7.8 delitos frente a los 3.9 del resto de pacientes, y presentan mayor tasa de consumo de drogas, desde edades más tempranas y en cantidades más elevadas.

La importancia referida al TDAH tanto como trastorno como por su impacto en la vida adulta, al menos por su prevalencia en prisión, haría suponer que se cuenta al menos con instrumentos de medida propios o adaptados a este ámbito. De nuevo el análisis bibliométrico utilizando como palabras clave 'ADHD', 'prisoners' y 'assessment', constata una realidad contundente: solamente dos artículos en revistas científicas (2011 y 2013) tratan este tema, mientras que cuando la búsqueda se cambia a 'assessment' por 'diagnostics' aglutina únicamente 5 artículos en revistas científicas desde 2005. El análisis constata un bajo nivel de interés en la evaluación de este trastorno en población reclusa, así como la ausencia de escalas específicas para la evaluación del TDAH en este entorno, si bien se tiende a adaptar aquellas utilizadas para evaluar el trastorno en adultos (Rösler et al., 2006). En este sentido, y centrado en el análisis de la prevalencia, destacan los trabajos realizados por Einarsson, Sigurdsson, Gudjonsson, Newton, y Bragason (2009) y Vegue-González et al. (2011) empleando la Wender Utah Rating Scale (WURS; Ward, Wender, \& Reimherr, 1993), así como los estudios llevados a cabo por Stokkeland, Fasmer, Waage, y Hansen (2014) o Usher, Stewart, y Wilton (2013) con la Adult ADHD Self-Report Scale (ASRS; Kessler et al., 2005).

En estos estudios se obtuvieron rangos de prevalencia para el TDAH en población reclusa que van desde 16 a $40 \%$. Señalando, además, que el TDAH se relaciona con 
inestabilidad laboral y altas tasas de reincidencia, con dificultades del aprendizaje durante la infancia y trastornos mentales durante la adolescencia y edad adulta, como trastorno de conducta antisocial, personalidad límite y abuso de sustancias entre otras.

Desde esta realidad, se hace necesario por tanto ampliar el estudio de la prevalencia del TDAH, pero también de sus consecuencias en población reclusa. Dentro de un marco de estudio más amplio, los objetivos del estudio son estimar la prevalencia con cribado positivo del TDAH en población penitenciaria, y estudiar las características sociodemográficas relevantes asociadas a este trastorno, comparando y correlacionando dos escalas de identificación del TDAH ampliamente utilizadas en el ámbito clínico y validadas en adultos, concretamente la WURS (Ward et al., 1993) y ASRS (Kessler et al., 2005), anteriormente mencionadas.

\section{Método}

\section{Participantes}

El estudio se ha realizado con un total de 143 internos penitenciarios, que accedieron a participar en el estudio sobre TDAH y contestaron íntegramente todos los cuestionarios utilizados para establecer la prevalencia. Sus edades estaban comprendidas en un rango de edad entre los $18 \mathrm{y}$ los 69 años, $(M d n=36)$. El grupo de internos penitenciarios estuvo conformado por 133 hombres ( $M d n=35$ años) y 10 mujeres con un rango de edad menor entre 41 y 59 años $(M d n=41)$, ofreciendo diferencias estadísticamente significativas en el género entre hombres y mujeres, $\chi^{2}$ $(1)=107.931, p=.001$, así como para la variable edad, $t$ $(158)=45.532, p=.001$.

Instrumentos

Adult ADHD Self-Report Scale (ASRS). Se trata de un cuestionario diseñado para el cribado del TDAH en adultos (Kessler et al., 2005), utilizado en diferentes estudios internacionales con muestras variadas, incluso con internos penitenciarios (Usher et al., 2013). Consta de 6 ítems: cuatro evalúan síntomas de inatención y los dos restantes síntomas de hiperactividad. Cada ítem tiene cinco opciones de respuesta según la presencia del síntoma en los últimos seis meses, desde $0=$ 'nunca' hasta $4=$ 'muy a menudo'. La puntuación total tiene un rango de 0 a 24 puntos. La versión española de este test ha sido validada en pacientes ambulatorios de un servicio de psiquiatría. Los autores de la validación recomiendan un punto de corte de 12 puntos, en el que las propiedades psicométricas frente al diagnóstico clínico estandarizado (DSM-IV)- fueron: una sensibilidad del $96.7 \%$ y una especificidad del $91.1 \%$; un valor predictivo positivo del $91.6 \%$, y negativo del $96.5 \%$; un índice kappa de .78 y área bajo la curva de .94 (odds ratio $=297.3$, con un intervalo de confianza del $95 \%=$ 76.2-1.159 (Ramos-Quiroga et al., 2009). El coeficiente alfa de Cronbach obtenido en este estudio fue de .72 .

Wender Utah Rating Scale (WURS). Es un cuestionario retrospectivo que consta de 61 ítems que evalúan la frecuencia con que se presentaban ciertos síntomas del TDAH en la infancia (Ward et al., 1993). Es una escala tipo Likert con 5 alternativas de respuesta, de $0=$ "nada o ligeramente" a 4 = "mucho". En el presente estudio se utilizó la subescala ADHD-WURS (25 items), que proporciona una puntuación que varía entre 0 y 100 . El coeficiente alfa de Cronbach de la validación en castellano fue de .94 (Rodríguez-Jiménez et al., 2001).

Trabajos previos llevados a cabo con población universitaria han revelado una buena consistencia interna y fiabilidad test-retest al cabo de un mes de las dos formas de WURS (Rossini \& O’Connor, 1995). Así mismo, análisis factoriales llevados a cabo en otro estudio han revelado que la escala de 25 ítems se ajusta a un modelo de tres factores (atención, hiperactividad y impulsividad), explicando el $59.4 \%$ de la varianza (McCann, Schelle, Ward, \& Roy-Byme, 2000), y clasificando correctamente el $64.5 \%$ de los pacientes que solicitaron una evaluación para el TDAH.

Los autores señalaron que la especificidad de la escala era baja y un diagnóstico de TDAH con sólo la escala WURS podría ser engañoso debido a su carácter retrospectivo, y que esta escala evalúa los síntomas de la infancia desde el punto de vista de una persona adulta. La escala WURS es una de las más usadas para el diagnóstico de TDAH en adultos, siendo traducida y adaptada al francés (Bayle, Krebs, Martin, Bouvard, \& Wender, 2003), italiano (Fossati et al., 2001), español (Rodríguez-Jiménez et al., 2001) y alemán (Retz-Junginger et al., 2003). Además se han realizado estudios de validación con diferentes tipos de muestras. Este es el caso del estudio llevado a cabo por Rodríguez-Jiménez et al. (2001), con una muestra española de pacientes en tratamiento por problemas de adicción, y Öncü, Ölmez, y Şentürk (2005) con una muestra clínica de TDAH, depresión y trastorno bipolar. En ambos estudios se obtuvo una elevada fiabilidad en la escala (con un Alfa de Cronbach en torno a .94).

En el caso de la presente muestra, se realizó un análisis factorial exploratorio para la prueba WURS, empleando el método de componentes principales y la rotación Varimax. Los resultados indicaron la presencia de 4 factores principales (impulsividad, problemas emocionales, déficit de atención, y un factor mixto), que explicaron el 59\% de la varianza. Este último factor estuvo formado por únicamente tres ítems, con pesos factoriales relativamente bajos (en torno a .40). El coeficiente de fiabilidad alfa de Cronbach obtenido en este estudio fue de .95 .

\section{Procedimiento}

El estudio se desarrolló de acuerdo al código ético de la Declaración de Helsinki y de la World Medical Association (Williams 2008), que refleja los principios éticos de la investigación realizada con humanos, contando con la aprobación del comité de ética de la Universidad. Todos los internos penitenciarios participaron en el estudio de manera voluntaria y anónima. 
Pérez, C. R., Pérez, J. C. N., Díaz, F. J. R., Granda, A. P., Molleda C. B. \& Fernández, T. G. (2015). Trastorno por Déficit de Atención e Hiperactividad (TDAH): Prevalencia y Características Sociodemográficas en Población Reclusa.

Previamente al contacto con los presos y a la aplicación de las pruebas relacionadas con el TDAH, se realizó la recogida de información disponible a través de los expedientes facilitados por la institución, de acuerdo con la Ficha de Historia Penitenciaria (Estrada, Rodríguez, \& Solano, 2012). Esta información fue empleada para establecer las características contextuales básicas de los individuos que han participado en el estudio, en tanto responde a las principales variables criminógenas de los internados en Centros Penitenciarios: Estado Civil, identifica el estado actual de convivencia de la pareja (casado, soltero o unión libre); Escolaridad y Actividad Laboral, se refiere al nivel de estudios (analfabeta, lee y escribe, estudios primarios, secundaria, bachillerato, universitarios y otros); Actividad laboral, tanto en la actualidad -actividad en la economía formal e informal- como en el desarrollo de su historia penitenciaria. Durante este estudio se utilizaron los datos relativos a la edad, sexo, estado civil, clase social percibida, situación laboral y escolaridad.

\section{Análisis de Datos}

El programa SPSS v.19 (Arbuckle, 2010) fue usado para llevar a cabo los análisis estadísticos correspondientes. El nivel de significación estadística se estableció en un valor de $p<.05$.

En primer lugar se comprobaron los principios de normalidad y distribución de las variables continuas. Se siguió para ello el criterio propuesto por Finney y DiStefano (2006), según el cual $\pm 2 \mathrm{y} \pm 7$ son los valores máximos aceptables en asimetría y curtosis para llevar a cabo análisis paramétricos. Además se comprobó la homocedasticidad de las puntuaciones y así determinar la homogeneidad de la varianza-covarianza con la prueba de $M$ de Bartlett-Box.

Previamente se realizó un análisis factorial exploratorio en la escala WURS (25 items) para comprobar su estructura en una muestra específica de carcelarios.

Seguidamente, se analizó la correlación entre las puntuaciones directas en las escalas WURS y ASRS, así como la capacidad predictiva la primera (de carácter retrospectivo) sobre la segunda (diagnóstico del TDAH en edad adulta). Para cumplir con este último objetivo se realizó un análisis de regresión lineal.

Según los resultados globales de la ASRS y del WURS se determinó la prevalencia 'aparente' de TDAH con cribado positivo (número de individuos con ASRS + o WURS+ dividido entre el número total de individuos de la muestra).

Tabla 1

Estadísticos Descriptivos de las Variables Continuas (puntuaciones directas en las escalas WURS y ASRS)

\begin{tabular}{ccccccc}
\hline & Mínimo & Máximo & $M$ & $S D$ & Asimetría & Curtosis \\
\hline WURS & 5 & 77 & 34.38 & 18.069 & .502 & -.640 \\
ASRS & 0 & 22 & 9.37 & 4.148 & .292 & -.031 \\
\hline
\end{tabular}

Notas. WURS $=$ Wender Utah Rating Scale; ASRS = Adult ADHD Self Report Scale; $M=$ media, $S D=$ desviación tìpica
A partir de estos resultados, y considerando los valores conocidos de sensibilidad y especificidad de la prueba, se estimó la prevalencia 'real', utilizando la siguiente fórmula (Aragonès, Cañisá, Caballero, \& Piñol-Moreso, 2013):

$$
p=(t+\beta-1) /(\alpha+\beta-1)
$$

Donde $p$ es prevalencia 'real', $t$ es la prevalencia 'aparente', $\beta$ es la especificidad y $\alpha$ es la sensibilidad. Para calcular este parámetro y su intervalo de confianza, se usó el programa EpiTools Epidemiological Calculators (Sergeant, 2013). Con objeto de determinar las características asociadas a los pacientes con cribado positivo para el TDAH, se formaron dos grupos de individuos según el resultado de la ASRS (cribado positivo con $\geq 12$ puntos y cribado negativo con $<12$ puntos), y del WURS (resultado positivo con $\geq 46$ puntos y cribado negativo con $<$ 46 puntos) y se compararon sus características utilizando ANOVA para variables continuas y el test de $\chi^{2}-\mathrm{o}$ el test exacto de Fisher- para las variables categóricas. El estadístico $Z$ se empleó para analizar las diferencias en porcentajes.

\section{Resultados}

La Tabla 1 muestra los estadísticos descriptivos para las puntuaciones directas en las escalas WURS y ASRS, cuyos valores de asimetría y curtosis se encuentran dentro de los límites de normalidad aceptados para llevar a cabo análisis paramétricos según MLG. Asimismo, para determinar la homogeneidad de la varianza-covarianza (también llamado homocedasticidad) se realizó la prueba de $M$ de Bartlett-Box obteniéndose resultados significativos, $M$ de Box $=5.544 ; F(1)=5.498, p=.019$.

En segundo lugar, cabe señalar la presencia de una alta y estadísticamente significativa correlación $(r$ Pearson $=$ $.433 ; p<.001$ ) entre las dos escalas empleadas en el diagnóstico del TDAH, existiendo una capacidad predictiva significativa de la escala WURS (de carácter retrospectivo) en los síntomas del TDAH en la infancia sobre la escala ASRS, empleada para el diagnóstico en adultos, $F(1,141)$ $=32.073, p<.001$. La puntuación en la primera escala explica más del 18 por ciento de la varianza en la escala ASRS $\left(R^{2}=.186\right)$. Los Coeficientes no estandarizados Beta $\left(\beta_{k}\right)$ fueron de 6.086 (Error típico $=.668, t=9.110$, $p<.001$ ) para la Constante y .097 (Error típico $=.017, t=$ $5.663, p<.001)$ para la escala WURS. 
Proporción de Cribados Positivos y Estimación de la Prevalencia según ASRS

Un total de 46 de los 143 reclusos obtuvieron una puntuación $\geq 12$ puntos en la ASRS, lo que representa una proporción del 32.2\% (IC 95\% =+/-11.16\%). A partir de este resultado, y considerando los datos de sensibilidad y especificidad de la ASRS, la estimación de la prevalencia 'real' fue del $26.5 \%$ (IC 95\% = +/-10.54\%). El valor predictivo de un resultado positivo fue del $79.7 \%$, y el valor predictivo negativo, del 98.7\% (fiabilidad $=93.0 \% ; J$ de Youden $=$ $87.8 \%$ ). En las Tablas 1 y 2 se muestran las características sociodemográficas de los participantes según la prevalencia de TDAH en la escala ASRS. Destacan como diferencias estadísticamente significativas las diferencias en porcentajes en el número de divorciados $(\mathrm{ASRS}+=13.04$ frente a ASRS- $=6.18 \%$ ), y en la situación laboral en cuando al porcentaje de desempleados (ASRS $+=50$ frente a ASRS$=35.05 \%$ ), que se revierte para el porcentaje de carcelarios con empleo $(\mathrm{ASRS}+=23.91$ frente a ASRS-=39.17\%). En el apartado de escolaridad es donde las diferencias son más nítidas; en cuanto a la proporción de internos con estudios primarios las diferencias son significativas (ASRS $+=21.73$ frente a ASRS- $=35.05 \%$ ). Obteniendo un mayor efecto estadístico ( $p \leq .001)$, el porcentaje de los que no acabaron secundaria (ASRS+=36.95 frente a ASRS $-=16.49 \%$ ). Además ninguno de los reclusos con ASRS+ accedió a estudios universitarios. Para la variable edad, el grupo con ASRS + presentaba una media de $32.51(S D=9.56)$, y el grupo ASRS- de $37.08(S D=10,81)[F(1,141)=4.890, p$ $\left.=.029, \eta_{\mathrm{p}}^{2}=.043\right]$.

Tabla 2

Características de los Carcelarios por Sexo y Nivel de Estudios según el Resultado de la ASRS

\begin{tabular}{|c|c|c|c|c|c|c|}
\hline & & $\begin{array}{c}\text { ASRS+ } \\
(n=46)\end{array}$ & $\begin{array}{l}\text { ASRS- } \\
(n=97)\end{array}$ & $S E$ & $Z$ score & $p$ \\
\hline \multirow{2}{*}{ Sexo } & Masculino & $43(93.47 \%)$ & $90(92.78 \%)$ & .045 & .152 & $n s$ \\
\hline & Femenino & $3(6.53 \%)$ & $7(7.22 \%)$ & .045 & -.152 & $n s$ \\
\hline \multirow{7}{*}{$\begin{array}{l}\text { Nivel de } \\
\text { estudios }\end{array}$} & Analfabeto/a & $1(2.17 \%)$ & $3(3.09 \%)$ & .029 & -.311 & $n s$ \\
\hline & Primaria & $10(21.73 \%)$ & $34(35.05 \%)$ & .082 & -1.611 & $*$ \\
\hline & Secundaria & $13(28.26 \%)$ & $21(21.64 \%)$ & .076 & .867 & $n s$ \\
\hline & Abandono universidad & $2(4.34 \%)$ & $6(6.18 \%)$ & .041 & -.446 & $n s$ \\
\hline & Universitarios & $0(0 \%)$ & $7(7.21 \%)$ & .038 & -1.868 & $*$ \\
\hline & No finaliza primaria & $3(6.52 \%)$ & $10(10.30 \%)$ & .051 & -.735 & $n s$ \\
\hline & No finaliza secundaria & $17(36.95 \%)$ & $16(16.49 \%)$ & .075 & 2.712 & $* *$ \\
\hline
\end{tabular}

Notas. ASRS $+=$ ASRS $\geq 12$ puntos; ASRS- $=$ ASRS $<12 ; S E=$ error estándar; $n s=$ diferencia estadísticamente no significativa. $* p \leq .05, * * p \leq .001$.

Proporción de Cribados Positivos y Estimación de la Prevalencia según WURS

En el caso de la escala Wender Utah Rating Scale (WURS), de los 143 reclusos evaluados, un total de 43 obtuvieron una puntuación $\geq 46$ puntos (criterio más restrictivo), lo que representa una proporción del $30.1 \%$ (IC 95\% = +/-11.51\%; ver Tablas 3 y 4). A partir de este resultado, y considerando los datos de sensibilidad y especificidad del WURS, la estimación de la prevalencia 'real' fue del $25.4 \%$ (IC 95\% = +/-10.92\%). El valor predictivo de un resultado positivo fue del $77.2 \%$, y el valor predictivo negativo del $96.9 \%$ (fiabilidad $=90.9 \% ; J$ de
Youden $=82.3 \%$ ). Para la variable edad, el grupo con WURS+ presentaba una media de $31.60(S D=8.76)$, y el grupo WURS- de $37.00(S D=10.76)[F(1,141)=6.738$, $\left.p=.011, \eta_{p}^{2}=.058\right]$.

Al igual que en la escala ASRS, en cuanto a las variables sociodemográficas, se encontraron diferencias estadísticamente significativas en el porcentaje de reclusos que no acabaron secundaria (WURS $+=30.23 \%$ frente a WURS - =20\%). En las Tablas 3 y 4 se muestran las características sociodemográficas de los participantes y sus porcentajes en función de la puntuación en la escala WURS. 
Pérez, C. R., Pérez, J. C. N., Díaz, F. J. R., Granda, A. P., Molleda C. B. \& Fernández, T. G. (2015). Trastorno por Déficit de Atención e Hiperactividad (TDAH): Prevalencia y Características Sociodemográficas en Población Reclusa.

Tabla 3

Características de los Carcelarios por Estado Civil, Clase Social y Situación Laboral según el Resultado de la ASRS

\begin{tabular}{|c|c|c|c|c|c|c|}
\hline & & $\begin{array}{c}\text { ASRS+ } \\
(n=46)\end{array}$ & $\begin{array}{l}\text { ASRS- } \\
(n=97)\end{array}$ & $S E$ & Zscore & $p$ \\
\hline \multirow{6}{*}{$\begin{array}{l}\text { Estado } \\
\text { Civil }\end{array}$} & Soltero/a & $27(58.69 \%)$ & $55(56.70 \%)$ & .088 & .225 & $n s$ \\
\hline & Casado/a & $4(8.69 \%)$ & $16(16.49 \%)$ & .062 & -1.256 & $n s$ \\
\hline & Divorciado/a & $6(13.04 \%)$ & $6(6.18 \%)$ & .049 & 1.381 & $*$ \\
\hline & Separado/a & $7(15.21 \%)$ & $10(10.30 \%)$ & .057 & .847 & $n s$ \\
\hline & Viudo/a & $0(0 \%)$ & $2(2.06 \%)$ & .021 & -.980 & $n s$ \\
\hline & Convivencia pareja & $2(4.34 \%)$ & $8(8.24 \%)$ & .045 & -.854 & $n s$ \\
\hline \multirow{3}{*}{$\begin{array}{c}\text { Clase Social } \\
\text { percibida }\end{array}$} & Baja & $12(26.08 \%)$ & $24(24.74 \%)$ & .077 & .173 & $n s$ \\
\hline & Media & $30(65.21 \%)$ & $68(70.10 \%)$ & .083 & -.587 & $n s$ \\
\hline & Alta & $4(8.69 \%)$ & $5(5.15 \%)$ & .043 & .814 & $n s$ \\
\hline \multirow{5}{*}{$\begin{array}{c}\text { Situación } \\
\text { laboral }\end{array}$} & Desempleado/a & $23(50 \%)$ & $34(35.05 \%)$ & .087 & 1.705 & $*$ \\
\hline & Empleado/a & $11(23.91 \%)$ & $38(39.17 \%)$ & .084 & -1.796 & $*$ \\
\hline & Economía informal & $1(2.17 \%)$ & $5(5.15 \%)$ & .035 & -.830 & $n s$ \\
\hline & Negocio propio & $5(10.87 \%)$ & $11(11.34 \%)$ & .056 & -.083 & $n s$ \\
\hline & Otros & $6(13.04 \%)$ & $9(9.27 \%)$ & .054 & .686 & $n s$ \\
\hline
\end{tabular}

Notas. ASRS $+=$ ASRS $\geq 12$ puntos; ASRS- $=$ ASRS $<12 ; S E=$ error estándar; $n s=$ diferencia estadísticamente no significativa. $* p \leq .05$.

Tabla 4

Características de los Carcelarios por Sexo y Nivel de Estudios según el Resultado del WURS

\begin{tabular}{lcccccc}
\hline \multirow{2}{*}{ Sexo } & & $\begin{array}{c}\text { WURS }+ \\
(n=43)\end{array}$ & $\begin{array}{c}\text { WURS- } \\
(n=100)\end{array}$ & SE & Z score & $p$ \\
& Masculino & $42(97.67 \%)$ & $91(91 \%)$ & .048 & 1.579 & $*$ \\
& Femenino & $1(2.33 \%)$ & $9(9 \%)$ & .048 & -1.579 & $*$ \\
& & & & & \\
& Analfabeto/a & $2(4.65 \%)$ & $2(2 \%)$ & .030 & .881 & $n s$ \\
& Primaria & $14(32.55 \%)$ & $31(31 \%)$ & .084 & .183 & $n s$ \\
& Secundaria & $9(20.93 \%)$ & $25(25 \%)$ & .077 & -.524 & $n s$ \\
Nivel de estudios & Abandono universidad & $0(0 \%)$ & $8(8 \%)$ & .041 & -1.908 & $*$ \\
& Universitarios & $0(0 \%)$ & $6(6 \%)$ & .036 & -1.641 & $*$ \\
& No finaliza primaria & $5(11.62 \%)$ & $8(8 \%)$ & .052 & .692 & $n s$ \\
& No finaliza secundaria & $13(30.23 \%)$ & $20(20 \%)$ & .076 & 1.331 & $*$ \\
\hline
\end{tabular}

Notas. WURS $+=$ WURS $\geq 46$ puntos; WURS- $=$ WURS $<46 ; S E=$ error estándar; $n s=$ diferencia estadísticamente no significativa. $* p \leq .05$. 
Tabla 5

Características de los Carcelarios por Estado Civil, Clase Social y Situación Laboral según el Resultado del WURS

\begin{tabular}{|c|c|c|c|c|c|c|}
\hline & & $\begin{array}{c}\text { WURS+ } \\
(n=43)\end{array}$ & $\begin{array}{l}\text { WURS- } \\
(n=100)\end{array}$ & $S E$ & Z score & $p$ \\
\hline \multirow{6}{*}{ Estado Civil } & Soltero/a & $27(62.79 \%)$ & $54(54 \%)$ & .090 & .972 & $n s$ \\
\hline & Casado/a & $4(9.30 \%)$ & $17(17 \%)$ & .064 & -1.195 & $n s$ \\
\hline & Divorciado/a & $4(9.30 \%)$ & $8(8 \%)$ & .050 & .257 & $n s$ \\
\hline & Separado/a & $5(11.62 \%)$ & $12(12 \%)$ & .059 & -.063 & $n s$ \\
\hline & Viudo/a & $0(0 \%)$ & $2(2 \%)$ & .021 & -.933 & $n s$ \\
\hline & Convivencia pareja & $3(6.97 \%)$ & $7(7 \%)$ & .046 & -.005 & $n s$ \\
\hline \multirow{3}{*}{$\begin{array}{l}\text { Clase Social } \\
\text { percibida }\end{array}$} & Baja & $11(25.58 \%)$ & $26(26 \%)$ & .079 & -.052 & $n s$ \\
\hline & Media & $29(67.44 \%)$ & $68(68 \%)$ & .085 & -.065 & $n s$ \\
\hline & Alta & $3(6.97 \%)$ & $6(6 \%)$ & .044 & .220 & $n s$ \\
\hline \multirow{5}{*}{ Situación laboral } & Desempleado/a & $15(34.88 \%)$ & $43(43 \%)$ & .089 & -.906 & $n s$ \\
\hline & Empleado/a & $16(37.20 \%)$ & $33(33 \%)$ & .086 & .486 & $n s$ \\
\hline & Economía informal & $0(0 \%)$ & $6(6 \%)$ & .036 & -.164 & $*$ \\
\hline & Negocio propio & $6(13.95 \%)$ & $10(10 \%)$ & .057 & .687 & $n s$ \\
\hline & Otros & $6(13.95 \%)$ & $8(8 \%)$ & .054 & 1.098 & $n s$ \\
\hline
\end{tabular}

Notas. WURS $+=$ WURS $\geq 46$ puntos; WURS- $=$ WURS $<46 ; S E=$ error estándar; $n s=$ diferencia estadísticamente no significativa. $* p \leq .05$.

\section{Discusión y Conclusiones}

Los datos obtenidos responden al objetivo de estimar la prevalencia del TDAH en población penitenciaria, estudiando la relación de éste con características sociodemográficas relevantes asociadas, empleando dos escalas ampliamente utilizadas en población adulta (ASRS y WURS).

Los resultados refieren una prevalencia del trastorno de TDAH para la población en prisión elevada (en torno al $25 \%$ ) y por encima de la estimada para la población en general, utilizando ambas escalas. De este modo, con una correlación alta y estadísticamente significativa entre las dos pruebas, uno de cada cuatro reclusos cumpliría los criterios del TDAH, que podría explicar y anticipar algunos de los aspectos relacionados con su conducta delictiva (Ramos-Quiroga et al., 2013). Estas cifras siguen la línea de los resultados encontrados en estudios previos empleando las mismas escalas, que situaban la prevalencia del trastorno entre el 16 y $40 \%$. Concretamente, son coincidentes el trabajo de Usher et al. (2013), los cuales encontraron una prevalencia para el TDAH del $25.2 \%$ en una muestra de 497 hombres internados en un centro penitenciario canadiense. Sin embargo, estas cifras se encuentran ligeramente por debajo de las obtenidas en otros estudios previos empleando otros instrumentos de medida, que situaban la prevalencia del trastorno en torno al $40 \%$ o incluso del 45 al $70 \%$ (Rösler et al., 2004). Es necesario tener en cuenta que en el presente estudio la tasa de prevalencia se calculó en sentido estricto, teniendo en consideración los datos de sensibilidad y especificidad de cada prueba para minimizar las limitaciones derivadas del uso de auto-informes (Ghanizadeh et al., 2011).

Por otro lado, se debe considerar que las consecuencias a largo plazo del TDAH en población penitenciaria no han sido ampliamente estudiadas, lo cual impone ciertas limitaciones que hemos intentado reducir en ese estudio. En este sentido, se encuentran tasas de prevalencia del TDAH muy altas y dispares, dependiendo del estudio y del país. Así, por ejemplo, en Suecia y Alemania las tasas varían entre el 40 y el $45 \%$, siendo en el sexo femenino del 10\% (Ginsberg et al., 2010). En el presente estudio el número de mujeres que cumplían los criterios de TDAH en el ASRS fue de 3 de las 10 (30\%) y 1 en el WURS 
Pérez, C. R., Pérez, J. C. N., Díaz, F. J. R., Granda, A. P., Molleda C. B. \& Fernández, T. G. (2015). Trastorno por Déficit de Atención e Hiperactividad (TDAH): Prevalencia y Características Sociodemográficas en Población Reclusa.

(10\%). Los resultados de estudios previos son, no obstante, inconsistentes y los resultados obtenidos dependen en gran medida de las características propias del TDAH, su complejidad y comorbilidad (Ramos-Quiroga et al., 2013). En Noruega, por ejemplo, se llevó a cabo un estudio longitudinal en niños con TDAH, observándose que el $27 \%$ de ellos fueron encarcelados de adultos. No obstante, se concluyó que los sujetos con TDAH en ausencia de Trastorno de Conducta en la infancia tienen el mismo riesgo que el resto de la población de cometer un delito en la edad adulta (Mordre, Groholt, Kjelsberg, Sandstad, \& Myhre, 2011). Estos resultados muestran que la cuestión sobre la relación entre TDAH y criminalidad y sus causas no está resuelta y que es necesario profundizar en ello (RamosQuiroga et al., 2013).

En este contexto, la asociación entre TDAH y la comisión de delitos que llevan a prisión puede deberse a dos factores. La primera hipótesis es que el TDAH puede directamente causar los comportamientos infractores. $\mathrm{La}$ segunda puede estar relacionada con el frecuente solapamiento entre TDAH y los trastornos de conducta. Para entender este planteamiento es necesario el análisis de las variables sociodemográficas de este grupo de forma retrospectiva, ya que el TDAH en la edad adulta afecta a las actuaciones educativas y ocupacionales, así como el funcionamiento psicológico y social, con un mayor riesgo de desempleo, bajas por enfermedad, problemas de pareja, trastornos coexistentes, o conducta antisocial entre otros (Ginsberg et al., 2010). En este estudio se ha comprobado que, en la población reclusa, el TDAH puede explicar diferencias en cuanto a Estado Civil, Clase Social percibida, Situación laboral, y Escolaridad. Los resultados obtenidos en el presente estudio evidencian la existencia de diferencias significativas entre los sujetos que cumplen los criterios para el TDAH y los que no, y con ciertas diferencias entre las dos pruebas utilizadas. Así por ejemplo, el alto porcentaje de divorciados que cumplen los criterios del TDAH en el ASRS es el doble que los que no lo cumplen. Aunque no significativa, también cabe destacar que la proporción de reclusos casados que no cumplen los criterios frente a los reclusos con TDAH es más del doble.

En cuanto a la clase social percibida, no existen diferencias significativas y mayoritariamente ambos grupos se consideran de clase social media. Sin embargo, las diferencias más importantes se encuentran en torno a la situación laboral y la escolaridad de la población reclusa. Respecto a la primera, los resultados en el ASRS aportaron diferencias estadísticamente significativas en torno a dos variables complementarias. Mientras que un alto porcentaje (casi la mitad) de los reclusos que cumplen los criterios TDAH están desempleados, el porcentaje de los que tienen empleo es significativamente inferior. Está relación se invierte en el caso de los reclusos que no los cumplen, situándose la tasa de empleados en un porcentaje más alto que el de desempleados. Los resultados evidencian el riesgo de presentar dificultades laborales en adultos con TDAH, que son agravadas por la situación de encarcelamiento.

Respecto a la escolaridad, existen diversos estudios que relacionan las Dificultades de Aprendizaje con el TDAH (Mayes, Calhoun, \& Crowell, 2000). Los problemas educativos y escolares de los niños y adolescentes con TDAH son evidentes y ampliamente trabajados. Estas ideas se evidencian en este estudio cuando se analiza la escolaridad. Concretamente, de los reclusos que cumplen los criterios TDAH en el ASRS, un porcentaje pequeño tienen estudios primarios, siendo en todo caso inferior a los que no los cumplen. De forma significativa, y reflejado tanto en el ASRS como en el WURS, el abandono escolar se ve reflejado en secundaria, con un porcentaje muy alto, y estadísticamente mayor, de los reclusos que cumplen los criterios de TDAH abandonando la escolaridad en esta etapa. Finalmente, ninguno de los reclusos que cumplen los criterios TDAH pudo concluir algún estudio universitario, aunque varios que no los cumplen si obtuvieron una titulación superior.

En definitiva, aunque las consecuencias a largo plazo de presentar TDAH en la infancia no están estudiadas en profundidad, claramente se observan las consecuencias del trastorno en la edad adulta. Parece probado además, que sufrir este trastorno en la infancia, y debido presumiblemente a las dificultades en el control de impulsivos, aumenta la tasa de participación en actividades delictivas, el abuso de sustancias (Fletcher \& Wolfe, 2009), así como de problemas en las relaciones de pareja y la empleabilidad. Por lo tanto, se puede esperar que implementar intervenciones eficaces para tratar el TDAH en la infancia contribuya a disminuir el riesgo de sufrir problemas futuros en este sentido, lo que representa un desafío por sí mismo en el estudio del TDAH.

Aun considerando las posibles limitaciones en la metodología utilizada, estos resultados abogan a cerca de la utilidad del propósito y estudio de la prevalencia con cribado positivo del TDAH en población penitenciaria. Estas limitaciones, en primer lugar, se refieren a los instrumentos utilizados para identificar los casos posibles de TDAH, si bien han sido aplicados en el contexto penitenciario, estos estudios han sido realizados en poblaciones diferentes a la española (Stokkeland et al., 2014). Dados los resultados obtenidos en el presente estudio, se asume no obstante, que su calibración es aplicable a nuestros internos penitenciarios. En segundo lugar, una dificultad asociada a la muestra obtenida es la diferente proporción de hombres - mujeres, que si bien representa la realidad de nuestro medio penitenciario -representada por 9 a 1-, debe ser tenida en cuenta para la interpretación de los resultados obtenidos con este colectivo (Rösler, Retz, Yaqoobi, Burg, \& Retz-Junginger, 2009).

En definitiva, los resultados obtenidos en este estudio indicarían la necesidad de estudiar las consecuencias a largo plazo de este trastorno en el contexto penitenciario, con el objeto de mejorar el manejo terapéutico de esta población. A su vez, se ofrece la oportunidad de responder 
a la necesidad de adaptar instrumentos de evaluación aplicables a diferentes ámbitos y poblaciones, en este caso al contexto penitenciario. En el caso de la presente estudio, el análisis factorial exploratorio, confirma resultados anteriores con algunas diferencias en uno de los factores, lo que plantea la cuestión acerca de su pertinencia en futuras versiones de la escala. No obstante, aunque los resultados de este estudio parecen consistentes, la ausencia de una validación exhaustiva de los instrumentos utilizados en poblaciones con reclusos sugiere extraer conclusiones con cierta cautela. Si bien los datos aportados por los análisis factoriales y de fiabilidad en base a la muestra del presente estudio invitan a ser optimistas respecto de las propiedades psicométricas de estas escalas con este tipo de poblaciones. Investigaciones futuras deberían abundar en su estudio.

\section{Referencias}

American Psychiatric Association. (2000). Diagnostic and statistical manual of mental disorders ( $4^{\text {th }}$ Rev. ed.). Washington, DC: Author.

Aragonès, E., Cañisá, A., Caballero, A., \& Piñol-Moreso, J. L. (2013). Cribado para el trastorno por déficit de atención/hiperactividad en pacientes adultos de atención primaria. Revista de Neurología, 56, 449-455.

Arbuckle, J. L. (2010). SPSS (Version 19.0) [Computer software]. Chicago, IL: Statistical Package for the Social Sciences.

Bayle, F. J., Krebs, M. O., Martin, C., Bouvard, M. P., \& Wender, P. (2003). French version of Wender Utah Rating Scale (WURS). Canadian Journal of Psychiatry, 48, 132.

Brown, R. T. (2003). Trastornos por déficit de atención y comorbilidades en niños, adolescentes y adultos. Barcelona, España: Masson.

Brown, R. T., Freeman, W. S., Perrin, J. M., Stein, M. T., Amler, R. W., Feldman, H. M., ...Wolraich, M. L. (2001). Prevalence and assesment of attention-deficit/hiperactivity disorder in primary care settings. Pediatrics, 107, 47-53. doi:10.1542/ peds.107.3.e43

Caballo, V. E., \& Simón, M. A. (2001). Manual de Psicología Clínica infantil y del adolescente: trastornos generales. Madrid, España: Pirámide.

Einarsson, E., Sigurdsson, J. F., Gudjonsson, G. H., Newton, A. K., \& Bragason, O. O. (2009). Screening for attention-deficit hyperactivity disorder and co-morbid mental disorders among prison inmates. Nordic Journal of Psychiatry, 63, 361-367. doi:10.1080/08039480902759184

Einat, T., \& Einat, A. (2008). Learning disabilities and delinquency. A study of Israeli Prison Inmates. International Journal of Offender Therapy and Comparative Criminology, 52, 416-434. doi:10.1177/0306624X07307352

Eme, R. (2008). Attention deficit/hyperactivity disorder and the juvenile justice system. Journal of Forensic Psychology Practice, 8, 174-185. doi:10.1080/15228930801963994

Estrada, C., Rodríguez, F. J., \& Solano, C. (2012). Castigo y carrera delictiva de liberados del sistema penitenciario de Jalisco. In S. P. Colín \& E. García (Eds.), Más allá de la violencia. Retos hacia la reconstrucción (pp. 291-320). Morelia, México: Universidad de Morelia.

Fazel, S., \& Seewald, K. (2012). Severe mental illness in 33.588 prisoners worldwide: Systematic review and meta-regression analysis. British Journal of Psychiatry, 200, 364-373. doi:10.1192/bjp.bp.111.096370
Finney, S. J., \& DiStefano, C. (2006). Non-normal and categorical data in structural equation modeling. In G. R. Hancock \& R. O. Muller (Eds.), Structural equation modeling: A second course (pp. 269-314). Greenwich, CT: Information Age.

Fletcher, J., \& Wolfe, B. (2009). Long-term consequences of childhood ADHD on criminal activities. Journal of Mental Health Policy and Economics, 12, 119-138.

Fossati, A., DiCeglie, A., Acquarini, E., Donati, D., Donini, M., Novella, L., \& Maffei, C. (2001). The retrospective assessment of childhood attention deficit hyperactivity disorder in adults: Reliability and validity of the Italian version of the Wender Utah Rating Scale. Comprehensive Psychiatry, 42, 326-336. doi:10.1053/comp.2001.24584

Ghanizadeh, A., Mohammadi, M., Akhondzadeh, S., \& SanaeiZadeh, H. (2011). Attention deficit hyperactivity disorder in imprisoned individuals - A review. Psychiatria Danubina, 23, 139-144.

Ghanizadeh, A., Nouri, S. Z., \& Nabr, S. S. (2012). Psychiatric problems and suicidal behavior in incarcerated adolescents in the Islamic Republic of Iran. Eastern Mediterranean Health Journal, 18, 311-317.

Ginsberg, Y., Hirvikoski, T., \& Lindefors, N. (2010). Attention deficit hyperactivity disorder (ADHD) among longer-term prison inmates is a prevalent, persistent and disabling disorder. BMC Psychiatry, 10, 112. doi:10.1186/1471-244X-10-112

Kessler, R., Adler, L., Ames, M., Demler, O., Faraone, S., Hiripi, E., ...Walters, E. (2005). The World Health Organization Adult ADHD Self-Report Scale (ASRS): A short screening scale for use in the general population. Psychological Medicine, 35, 245-256.

Martin, M. S. M., Wamboldt, A. D., O’Connor, S. L., Fortier, J., \& Simpson, A. (2013). A comparison of scoring models for computerised mental health screening for federal prison inmates. Criminal Behaviourand Mental Health, 23, 6-17. doi: $10.1002 / \mathrm{cbm} .1853$

Mayes, S. W., Calhoun, S. L., \& Crowell, E. W. (2000). Learning disabilities and ADHD: Overlapping spectrum disorders. Journal of Learning Disabilities, 33, 417-424.

McCann, B. S., Schelle, L., Ward, N., \& Roy-Byme, P. (2000). Discriminant validity of the Wender Utah Rating Scale for attention deficit hyperactivity disorder in adults. Journal of Neuropsychiatry and Clinical Neurosciences, 12, 240-245.

Mordre, M., Groholt, B., Kjelsberg, E., Sandstad, B., \& Myhre, A. M. (2011). The impact of ADHD and conduct disorder in childhood on adult delinquency: A 30 years follow-up study using official crime records. BMC Psychiatry, 11, 57. doi:10.1186/1471-244X-11-57

Öncü, B., Ölmez, S., \& Şentürk, V. (2005). Validity and reliability of Turkish version of Wender-Utah Rating Scale for attention deficit hyperactivity disorder in adults. Turkish Journal of Psychiatry, 16(4), 252-259.

Ramos-Quiroga, J. A., Daigre, C., Valero, S., Bosch, R., GómezBarros, N., Nogueira, M., ...Casas, M. (2009). Validación al español de la escala de cribado del trastorno por déficit de atención/hiperactividad en adultos (ASRS v. 1.1): una nueva estrategia de puntuación. Revista de Neurología, 48, 449-452.

Ramos-Quiroga, J. A., Montoya, A., Kutzelnigg, A., Deberdt, W., \& Sobanski, E. (2013). Attention deficit hyperactivity disorder in the European adult population: Prevalence, disease awareness, and treatment guidelines. Current Medical Research and Opinion, 29, 1093-1104.doi: 10.1185/03007995.2013.812961 Retz-Junginger, P., Retz, W., Blocher, D., Stieglitz, R. D., Georg, T., Supprian, T., ...Rösler, M. (2003). Reliability and validity 
Pérez, C. R., Pérez, J. C. N., Díaz, F. J. R., Granda, A. P., Molleda C. B. \& Fernández, T. G. (2015). Trastorno por Déficit de Atención e Hiperactividad (TDAH): Prevalencia y Características Sociodemográficas en Población Reclusa.

of the Wender Utah Rating Scale short form. Retrospective assessment of symptoms for attention deficit/hyperactivity disorder. Nervenarzt, 74, 987-993.

Rodríguez-Jiménez, R., Ponce, G., Monasor, R., Jiménez-Giménez, M., Pérez Rojo, J. A., Rubio, G., ...Palomo, T. (2001). Validation in the adult Spanish population of the Wender Utah Rating Scale for the retrospective evaluation in adults of attention deficit/hyperactivity disorder in childhood. Revista de Neurología, 33, 138-144.

Rösler, M., Retz, W., Retz-Junginger, P., Hengesch, G., Schneider, M., Supprian, T., ...Thome, J. (2004). Prevalence of attention deficit-/hyperactivity disorder (ADHD) and comorbid disorders in young male prison inmates. European Archives of Psychiatry and Clinical Neuroscience, 254, 365-371.

Rösler, M., Retz, W., Thome, J., Schneider, M., Stieglitz, R. D., \& Falkai, P. (2006). Psychopathological rating scales for diagnostic use in adults with attention-deficit/hyperactivity disorder (ADHD). European Archives of Psychiatry and Clinical Neuroscience, 256, 3-11.

Rösler, M., Retz, W., Yaqoobi, K., Burg, E., \& Retz-Junginger, P. (2009). Attention deficit/hyperactivity disorder in female offenders: Prevalence, psychiatric comorbidity and psychosocial implications. European Archives of Psychiatry and Clinical Neuroscience, 259, 98-105.

Rossini, E. D., \& O'Connor, M. A. (1995). Retrospective selfreported symptoms of attention deficit hyperactivity disorder reliability of the Wender Utah Rating Scale. Psychological Repiorts, 77, 751-754.

Sanz-García, O., Dueñas, R. M., \& Muro, A. (2010). Consecuencias del TDAH no tratado: estudio en una población penitenciaria psiquiátrica. Psiquiatria.com. Recuperado en http://www.psiquiatria.com/tdah/consecuencias-del-tdah-notratado-estudio-en-una-poblacion-penitenciaria-psiquiatrica/

Schatz, D., \& Rostain, A. (2006). ADHD with comorbid anxiety: A review of the current literature. Journal of Attention Disorders, 10, 141-149.

Sergeant, E. S. G. (2013). Epitools epidemiological calculators. Retrieved from http://epitools.ausvet.com.au

Spencer, T., Abikof, H., Connor, D., Biederman, J., Pliszka, S. R., Boellner, S., ...Pratt, R. (2006). Efficacy and safety of mixed amphetamine salts extended release (Adderal XR) in the management of oppositional defiant disorder with or without comorbid attention-deficit/hyperactivity disorder. Clinical Therapeutics, 28, 402-418.

Stokkeland, L., Fasmer, O. B., Waage, L., \& Hansen, A. L. (2014). Attention deficit hyperactivity disorder among inmates in Bergen Prison. Scandinavian Journal of Psychology, 55, 343-349. doi:10.1111/sjop.12131

Usher, A. M., Stewart, L. A., \& Wilton, G. (2013). Attention deficit hyperactivity disorder in a Canadian prison population. International Journal of Law and Psychiatry, 36, 311-315. doi:10.1016/j.ijlp.2013.04.005

Vegue-González, M., Álvaro-Brun, E., Santiago-Sáez, A., \& Kanaan-Kanaan, A. (2011). Retrospective evaluation of attention deficit hyperactivity disorder with the Wender Utah Rating Scale in a sample of Spanish prison inmates. Journal of Forensic Sciences, 56, 1556-1561.doi:10.1111/j.15564029.2011.01895.x

Vicens-Pons, E., \& Grupo de Prevalencia en Cárceles. (2009). Aproximación a la metodología para el estudio de los trastornos mentales en población penitenciaria. El estudio PreCa Revista Española de Sanidad Penitenciaria, 11, 17-25.
Ward, M. F., Wender, P. H., \& Reimherr, F. W. (1993). The Wender Utah Rating Scale: An aid in the retrospective diagnosis of childhood attention deficit hyperactivity disorder. American Journal of Psychiatry, 150, 885-890.

Williams, J. R. (2008). Revising the Declaration of Helsinki. World Medical Journal, 54, 120-125.
Recebido: 04/11/2014

$1^{a}$ revisão: 27/02/2015

$2^{a}$ revisão: $28 / 05 / 2015$

Aceite final: 05/06/2015 